Rolf W. Huegli

Phillip F. J. Tirman

Harald M. Bonel

Harald Staedele

Souhil Zaim

Mikayel Grigorian

Harry K. Genant

\section{Use of the modified three-point Dixon technique in obtaining T1-weighted contrast-enhanced fat-saturated images on an open magnet}

Received: 13 October 2003

Revised: 5 March 2004

Accepted: 30 March 2004

Published online: 21 April 2004

(C) Springer-Verlag 2004

R. W. Huegli · H. M. Bonel · S. Zaim M. Grigorian · H. K. Genant

Osteoporosis and Arthritis Research Group, Department of Radiology,

University of California,

350 Parnassus Ave., Suite 150

San Francisco, CA 94117, USA

R. W. Huegli $(-$ H. Staedele

Departement Medizinische Radiologie, Kantonsspital Basel, Universitätskliniken, Petersgraben 4, 4031 Basel, Switzerland e-mail: rhuegli@uhbs.ch

Tel.: +41-61-2652525

Fax: +41-61-2655383

P. F. J. Tirman

National Orthopaedic Imaging Associates,

Marin Magnetic Imaging,

Greenbrae, CA 94904, USA

\begin{abstract}
The purpose of this study was to investigate the modified three-point Dixon technique as a method for obtaining fat-saturated T1-weighted sequences before and after intravenous gadolinium administration using an open MR imaging scanner. A preliminary experiment using an oil/gadolinium phantom was performed on a $0.35-\mathrm{T}$ open magnet and an advanced 1.5-T unit. Fat saturation was achieved at $1.5 \mathrm{~T}$ using a frequency selective presaturation technique and a modified three-point Dixon technique on the low-field scanner. The modified three-point Dixon sequence was then evaluated in ten patients undergoing MRI examinations of the spine with gadolinium enhancement to determine image characteristics and diagnostic potential. The phantom study demonstrated a homogenous suppression of signal from oil and a good distinction between fat and a
\end{abstract}

gadolinium chelate on the $0.35-\mathrm{T}$ unit comparable to that on the 1.5-T scanner. By applying the modified three-point Dixon technique on the open-magnet, the distinction between fat and gadolinium dimeglumine was rated as very good in 139 and good in 17 axial slices in a total of 156 images. No image was rated as difficult or not possible. Motion artifacts that hampered the reading were detected in the lower cervical spine due to respiratory movement in four (3\% of all) images. The modified three-point Dixon technique provides the combination of gadolinium enhancement with fat saturation on an open magnet. Early clinical applications appear promising.

Keywords Three-point Dixon technique - Fat suppression . Contrast-enhancement $\cdot$ MRI

\section{Introduction}

T1-weighted, contrast-enhanced, fat-suppressed imaging compared to non-fat-suppressed images is a well-established method to evaluate pathology made more conspicuous by contrast enhancement [1-5]. Open MR imaging is an emerging technology with certain advantages compared to a whole body scanner, especially for claustrophobic patients and MR interventions. Additionally, lowfield open MR imagers cost less than high-field superconducting magnets. One major drawback of these systems has been the inability to achieve T1-weighted fat- saturated imaging and therefore no reliable distinction between fat and contrast media [6].

Recently, a new technique has been developed using three-point Dixon chemical shift imaging tested in the musculoskeletal field for acute bone fractures [7] and cartilage evaluation in the knee joint [8]. These studies demonstrate that a reliable and homogeneous fat suppression is achievable on a $0.35-\mathrm{T}$ scanner.

This study was conducted to answer the following questions: is a distinction between fat and gadolinium possible in contrast-enhanced images on a 0.35-T lowfield scanner, how frequently do motion and phase arti- 
facts occur in examinations of the spine, and how useful is this sequence for routine application in a clinical setting?

\section{Materials and methods}

The modified three-point Dixon technique utilized in this study was supplied with a 0.35-T Toshiba Opart open magnet (Toshiba America MRI, South San Francisco, CA). The technique we used is based on the chemical shift imaging technique originally described by Dixon [9] and modified by Zhang in 1996 [10]. This imaging method can be applied to a low-field magnet, utilizing a single radiofrequency echo single-scan method for water and fat separation with correction of the static field inhomogeneities.

\section{Phantom measurements}

In a pilot study, test images were acquired using a liquid phantom consisting of an inner container filled with diluted gadopentate dimeglumine $\left(0.1 \mathrm{mmol} / \mathrm{kg} \mathrm{H}_{2} \mathrm{O}\right)$ surrounded by olive oil in an outer container. The phantom was placed in a commercially available standard head coil. The head coil as the most homogeneous coil was chosen in order to differentiate artifacts caused by shortcomings of the sequence and not by coil inhomogeneity.

The phantom measurements on the 0.35-T Toshiba Opart open magnet were performed using a conventional 2D T1-weighted spin-echo sequence [450/15 (repetition time in ms/echo time in $\mathrm{ms}$ ); section thickness, $4 \mathrm{~mm}$; number of signals acquired, 3; matrix, $256 \times 256$; field of view, $17 \times 12 \mathrm{~cm}$ ] and a T1-weighted $2 \mathrm{D}$ gradient-echo three-point Dixon study (water field echoes, WFE) for fat-saturated imaging $[750 / 20 / \pm 10.2$ (repetition time in $\mathrm{ms} / \mathrm{echo}$ time in $\mathrm{ms} / \tau$ in $\mathrm{ms}$ ); flip angle, $90^{\circ}$; section thickness, $4 \mathrm{~mm}$; number of signals acquired, 2; matrix, 256×176; field of view, $17.0 \times 12.8 \mathrm{~cm}]$. Likewise, on the 1.5 -Tesla unit (Signa, General Electric Medical Systems, Milwaukee, WI), spin-echo 2D T1weighted images [300/16 (repetition time in ms/echo time in $\mathrm{ms}$ ); section thickness, $4 \mathrm{~mm}$; number of signals acquired, 1 ; matrix, $256 \times 256$; field of view, $12 \times 12 \mathrm{~cm}]$ and fat-saturated $2 \mathrm{D} \mathrm{T} 1-$ weighted images using a conventional frequency selective fat saturation pulse [360/12 (repetition time in ms/echo time in ms); section thickness, $4 \mathrm{~mm}$; number of signals acquired, 1; matrix, $256 \times 160$; field of view, $12 \times 12 \mathrm{~cm}$ ] were obtained.

\section{Clinical MR examinations}

During a period of 6 months in 1999, 257 patients were referred to the open low-field magnet for an MRI examination of the spine, the majority with benign and chronic complaint. A total of ten patients (five men and five women, with an age range of 23-56 years and a median age of 48 years) formed our study population, and gadopentetate dimeglumine $(0.1 \mathrm{mmol} / \mathrm{kg}$ bw; Magnevist; Berlex Laboratories, Inc.; Montville, NJ) was administrated intravenously. Indications for the contrast-enhanced MR exams were suspected infection, bone marrow disease or persistent postoperative back pain. The cervical spine was scanned in three cases, and the lumbar spine in seven. Written informed consent was obtained from all patients prior to the MR exam. All clinical MR imaging examinations were performed on a Toshiba Opart 0.35-T open magnet.

Axial T1-weighted spin-echo images and fat-saturated images with the modified three-point Dixon method were obtained in addition to those acquired as part of the routine protocol. The modified three-point Dixon technique was added to gain contrastenhanced fat-saturated images.

Axial T1-weighted 2D gradient-echo three-point Dixon studies $[700-750 / 20 / \pm 10.2$ (repetition time in $\mathrm{ms} /$ echo time in $\mathrm{ms} / \tau$ in $\mathrm{ms}$ ); flip angle, $90^{\circ}$; section thickness, $4-5 \mathrm{~mm}$; number of signals acquired, $1-2$; matrix, $192 \times 256-256 \times 256$; field of view, $18 \times 18-$
$23 \times 23 \mathrm{~cm}$; no phase wrap to avoid aliasing] were obtained consecutively. The relatively long TR was chosen for the additional number of slices available in order to cover the anatomic region in the shortest time possible. The number of slices for a given TR utilizing the three-point Dixon technique is slightly reduced when compared to a conventional technique.

The bandwidth was $25 \mathrm{kHz}$. Adding three images together as in the three-point Dixon technique equate the water field echo sequence to correspond to a bandwidth of $8.5 \mathrm{kHz}$ compared to $10 \mathrm{kHz}$ for a non-fat-suppressed sequence. The acquisition time for the complete Dixon study of the lumbar or cervical spine lasted between 4.5 and $6.4 \mathrm{~min}$. Commercially available combined solenoidal and linear coils were utilized for the studies of the cervical and lumbar spine.

\section{Evaluation protocol}

Three experienced radiologists analyzed the axial slices of the modified three-point Dixon studies retrospectively in one session. The images of all studies were presented for each MR examination at the time of reading. A consensus reading was performed by all three radiologists. The fat-only images were not used at any stage of the diagnostic evaluation.

The following image characteristics were part of the evaluation: (1) the distinction between fat and gadolinium was rated on an ordinal scale as very good, good, difficult or not possible; (2) the frequency of motion and phase artifacts were documented as no displacement, minor displacement (no hampering of the read out) or major displacement resulting in an image quality below diagnostic requirements; (3) finally, a decision was made on the usefulness of routine reporting of the modified three-point Dixon sequence.

\section{Results}

\section{Phantom}

Figure 1 illustrates the results of the phantom measurements with the gadopentate dimeglumine container in the center surrounded by olive oil. The fat-saturated T1weighted image in Fig. 1a obtained with the modified three-point Dixon technique reveals a homogeneous fat suppression comparable in quality to the T1-weighted 2D image (Fig. 1b) obtained with the conventional presaturation technique on the high-field scanner.

The two thin but distinct crescent-shaped bright rings seen in the periphery surrounding the olive oil container on the modified three-point Dixon image represent a double vision artifact. The double vision artifact as a signal contamination is observed at the edge of the compartments because of a difference in geometric distortion between the in-phase RF echo image and the two out-ofphase gradient-echo images [10]. This artifact was not observed in any of our clinical studies, but typically occurs on sharp edges in the periphery of the image.

\section{Patients}

Distinction between fat and gadolinium. The distinction between fat and gadolinium dimeglumine was rated as very good in 139 (89\%) (Fig. 2) or good in 17 (11\%) 
Fig. 1 MR images of the gadolinium (inner container)/ oil (outer container) phantom acquired applying the WFE (gradient-echo) sandwich sequence for the fat separation images. a Modified three-point Dixon sequence on the $0.35-\mathrm{T}$ open magnet revealing a homogenous fat suppression comparable in quality to the fat-suppressed spin-echo sequence of a 1.5-T unit $\mathbf{b}$. Two thin, crescent-shaped, bright rings surround the olive oil container and represent a double vision artifact of the modified three-point Dixon image a

Fig. 2 Axial MR images of the cervical spine in a 23 -year-old patient with pain in the upper right neck (marker indicated by curved arrow). a Pre-contrast spin-echo T1-weighted image showing several round masses (solid arrows), b which are not suppressed in a STIR sequence. c The three-point Dixon image reveals a homogeneous fat suppression, and the left-sided enhancing mass representing swollen lymph nodes in the course of metastatic disease. Discrete susceptibility artifacts can be recognized on the air/soft tissue interface (arrowhead)
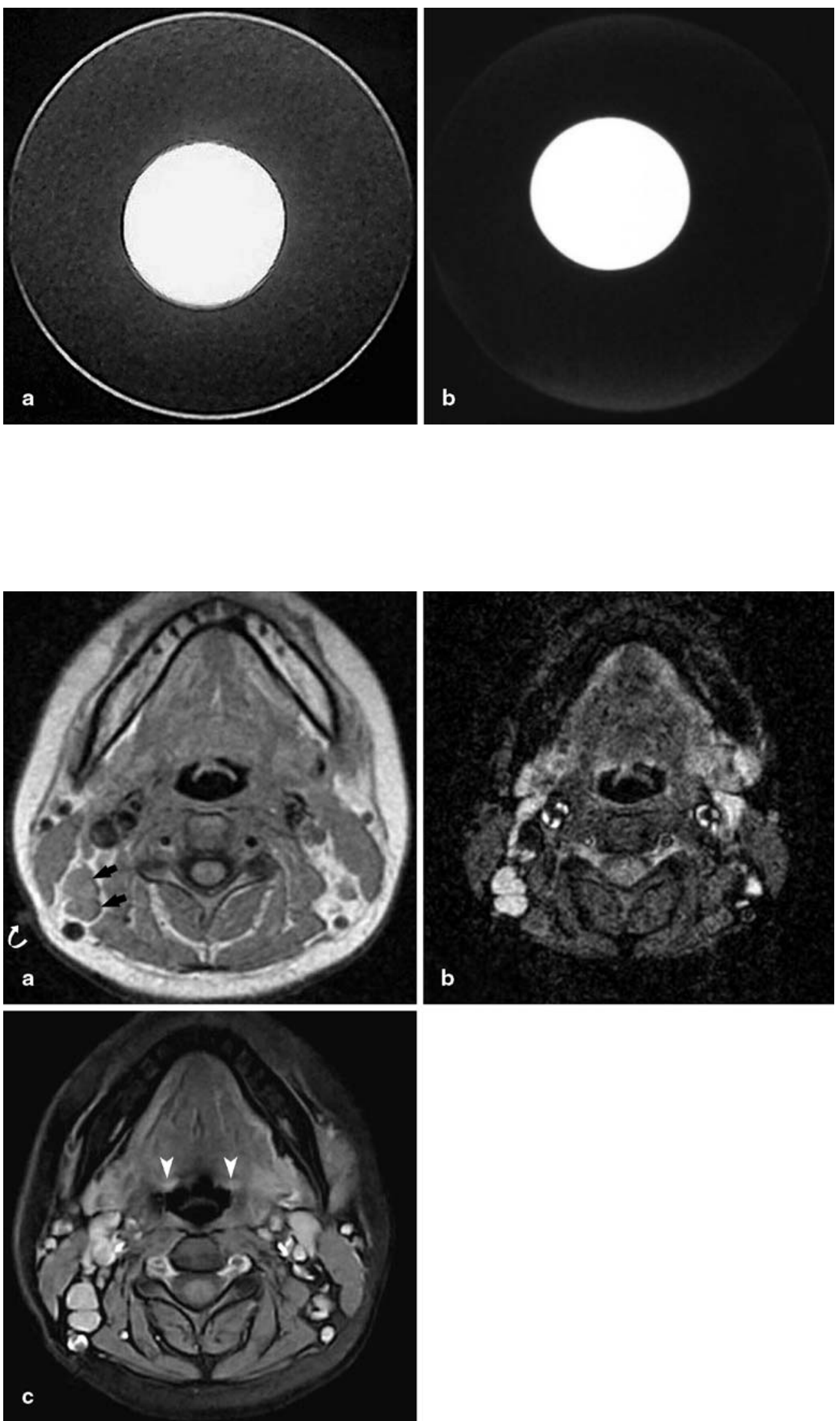


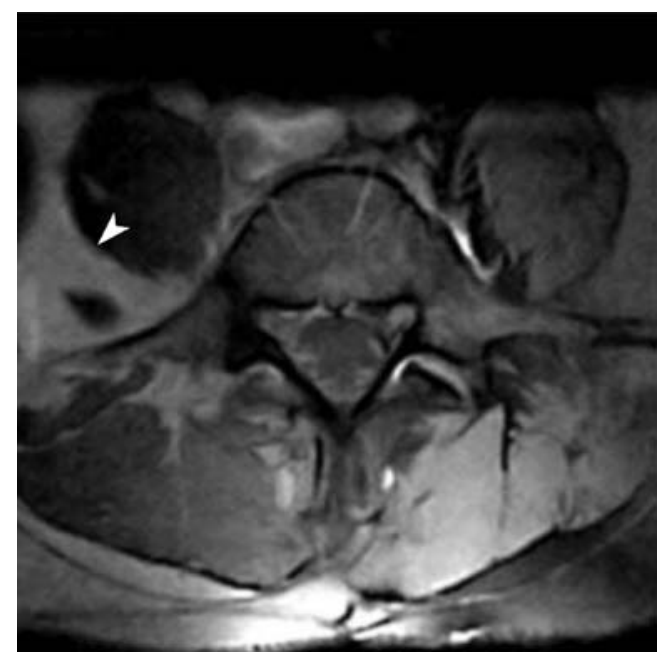

Fig. 3 Axial MR images of a lumbar spine demonstrating a fat suppression artifact in a 56-year-old patient without a pathological finding. Modified three-point Dixon sequence revealing a nonuniform fat suppression with a paradoxical water saturation in the right psoas muscle region (arrowhead). This artifact can be found on high-field and low-field scanners because the three-point Dixon technique is like the presaturation pulse technique on highfield magnets, a frequency-dependent fat saturation method relying on magnetic field homogeneity

axial slices (Fig. 3). No image was rated as difficult or not possible.

Frequency of motion and phase artifact. In 117 (75\%) images, displacement artifacts were completely absent. Minor displacement artifacts that did not hamper the reading occurred in 35 (22\%) images. Major displacement artifacts seen in four (3\%) images were due to chest movement in a lower cervical spine exam.

Usefulness for routine examination. Thirty-six scanned disc levels, out of a total of 37 covered in axial planes, were useful for routine reporting. The single unanalyzable scan was due to the chest movement artifact mentioned above.

\section{Discussion}

Suppression of the relatively high signal intensity of fat in T1-weighted images leads to a more efficient use of the dynamic range of gray levels used to display the tissue contrast of MR images enhanced by paramagnetic agents. Therefore, the conspicuity of enhancing tissues after injection of T1-shortening gadolinium chelates is increased on T1-weighted images by using fat suppression, because the images devoid of the high fat signal are rescaled [2-4].

The STIR and the frequency selective presaturation pulse techniques are routine sequences on every stan- dard, up-to-date high-field MR imaging unit and are effective fat suppression techniques [2, 11, 12]. Intravenous gadolinium offers an additional diagnostic advantage $[13,14]$. Because intravenous paramagnetic contrast material shortens the T1-relaxation of enhancing tissues to the same range as that of fat, the STIR sequence is not applicable in fat-suppressed contrastenhanced MR imaging, as fat and enhancing tissues are equally suppressed [15]. Also, average image signal is frequently small in comparison to the high noise levels in STIR imaging, which is a major drawback for the utility of this sequence in routine imaging.

Another fat suppression technique applied on highfield scanners is based on the chemical shift between water and fat using a frequency-selective presaturation technique $[11,12,16]$. The main magnetic field of lowfield open magnets is considerably poorer in homogeneity and uniformity than that of conventional superconductive MR scanners, particularly at the periphery of the gantry. The main obstacle to achieving an acceptable fat suppression on low-field magnets is the fact that the spectral separations between the fat and water proton pools are too narrow to allow selective excitation of a single resonance. Frequency-selective fat suppression techniques are therefore not applicable on a low-field open scanner [6].

An alternative approach is the chemical shift imaging as first introduced by Dixon. It offers the opportunity to apply appropriate post-processing, which results in contrast-enhanced fat-suppressed images even on a low-field open magnet [9]. In the original version, two images (two Point Dixon technique) were acquired with fat and water in-phase and out-of-phase, respectively.

In this study, we applied a single RF echo, single-scan method for water and fat separation first described by Zhang et al. [10]. By adding a third acquisition (in our study an out-of-phase image), off-resonance phase accumulations can be achieved on a pixel-by-pixel basis and provide the possibility to calculate a $B 0$ field map. This map is used to compensate for phase shifts caused by $B 0$ inhomogeneities in each pixel and therefore allows a more accurate decomposition into water-specific and fatspecific images [10].

Various three-point Dixon methods have been applied in a broad variety of clinical applications. One potential problem in previous studies was the relatively long acquisition time needed to acquire multiple in-phase and out-of-phase images [17]. The major advantage of the modified three-point Dixon sandwich technique applied in this study is the greatly reduced scan time. The use of a relatively long TR provided an acceptable trade-off between complete anatomic coverage and sufficient T1weighting. By combining three acquisitions in a single scan, the scan time of the "sandwich sequence" is only one-third of that of a single-echo method [18]. This is a very important feature in a clinical setting, where scan 
time is relevant from an economical point of view as well as for the patients' comfort. The sandwich technique is not as prone to image displacement artifacts as the multiple echo methods because of the shorter acquisition time of the complete scan (4.5-6.4 min in our study for a lumbar or cervical spine) and the shorter lag between the three acquisitions. We encountered major displacement artifacts that interfered with our reading in only four (3\% of all) images. This artifact on one single disc level was due to chest movement in a lower cervical spine exam. Movement artifacts, however, can significantly deteriorate the image quality of all MR sequences that are in routine use today. Still, in comparison to prior versions of the three-point Dixon sequence, the sandwich technique contributes to the reduction of movement artifacts by shortening the imaging time and the time lag between acquisitions of the multiple image layers.

The phantom measurements on the open low-field magnet (applying the modified three-point Dixon technique) revealed a very effective distinction between homogeneous fat suppression of the gadolinium chelate, comparable in quality to the presaturation pulse technique on the high-field unit. The chemical shift artifact in the frequency encoding direction was not as pronounced on the low-field unit as the high-field scanner. However, a double vision artifact (Fig. 1a) was noticed on the modified three-point Dixon image on the lowfield open scanner. This artifact was detected on the sharp edges at the periphery of the phantom and was caused by a difference in the geometric distortion between the in-of-phase and out-of-phase images. The artifact was not noted on any of the clinical images, even after a thorough review. An algorithm to correct the double vision artifact has been created [10] and is in the process of undergoing clinical validation for application to routine imaging.

The results of the present clinical study indicate that the modified three-point Dixon chemical shift imaging sandwich technique offers a good distinction between fat and paramagnetic contrast media in all exams of the cer- vical and lumbar spine. The capability of acquiring images with and without fat saturation before and after an intravenous gadolinium administration on a low-field open magnet is a valuable development. This practical new sequence might be an advantageous addition to existing imaging protocols in diagnostic situations in which the anatomic structures of interest are enhanced only modestly with paramagnetic contrast.

The proposed sequence may have implications on MR-guided interventional procedures, for example, for percutaneous abdominal and skeletal biopsies, which combine the advantages of an open magnet with the ability to apply contrast agents and fat saturation at lowfield strength [19].

Further studies using the modified three-point Dixon sandwich technique and its correction algorithms are necessary, and additional trials directly comparing the standard fat suppression techniques applied on high-field scanners should be performed. This study, however, shows that this new sequence is valuable for routine reporting and that further research should be pursued to improve this sequence type.

\section{Conclusion}

The modified three-point Dixon sandwich technique provides a method of gaining acceptable, fat-suppressed contrast-enhanced images of cervical and lumbar spine exams on a $0.35-\mathrm{T}$ open MR system. Combining three echoes in a single-scan offers the added advantages of acquiring fast and reliable contrast-enhanced fat-suppressed images even when $B 0$ homogeneity is limited. Displacement artifacts occur in a very small fraction of the images. The first clinical application appears promising.

Acknowledgments The authors would like to thank Weiguo Zhang, $\mathrm{PhD}$, and Yves Miaux, MD PhD, for their scientific support as well as Victoria Vandenberg for editing the final version of this paper.

\section{References}

1. Daniel BL, Butts K, Glover GH, Cooper C, Herfkens RJ (1998) Breast cancer: gadolinium-enhanced MR imaging with a $0.5-\mathrm{T}$ open imager and three-point Dixon technique. Radiology 207:183-190

2. Hirsch JA, Loevner LA, Yousem DM, Siegelman ES, Keiper MD, Marquis RP, Grossman RI (1998) Gadoliniumenhanced fat-suppressed T1-weighted imaging of the head and neck: comparison of gradient and conventional SE sequences. J Comput Assist Tomogr 22:771-776
3. Battolla L, Zampa V, Mascalchi M, Falaschi F, Bagnolesi P, Tessa C, Paolicchi A, Bartolozzi C (1994) Gadolinium-DTPA combined with a fat suppression technique in studies with magnetic resonance at $0.5 \mathrm{~T}$ in soft-tissue neoplasms. Radiol Med (Torino) 88:353358

4. Schreiber WG, Brix G, Knopp MV, Hess T, Lorenz WJ (1996) Improved visualization of breast lesions with gadolinium-enhanced magnetization transfer MR imaging. Magn Reson Med 35:861-869
5. Longo M, Granata F, Ricciardi K, Gaeta M, Blandino A (2003) Contrastenhanced MR imaging with fat suppression in adult-onset septic spondylodiscitis. Eur Radiol 13:626637

6. Peterfy CG, Roberts T, Genant HK (1998) Dedicated extremity MR imaging: an emerging technology. Magn Reson Imaging Clin N Am 6:849-870 
7. Wohlgemuth WA, Roemer FW, Bohndorf K (2002) Short tau inversion recovery and three-point Dixon waterfat separation sequences in acute traumatic bone fractures at open 0.35 tesla MRI. Skeletal Radiol 31:343-348

8. Bredella MA, Losasso C, Moelleken SC, Huegli RW, Genant HK, Tirman PF (2001) Three-point Dixon chemicalshift imaging for evaluating articular cartilage defects in the knee joint on a low-field-strength open magnet. AJR Am J Roentgenol 177:1371-1375

9. Dixon WT (1984) Simple proton spectroscopic imaging. Radiology 153:189-194

10. Zhang W, Goldhaber DM, Kramer DM (1996) Separation of water and fat MR images in a single scan at $0.35 \mathrm{~T}$ using "sandwich" echoes. J Magn Reson Imaging 6:909-917
11. Mao J, Yan H, Bidgood WD Jr (1992) Fat suppression with an improved selective presaturation pulse. Magn Reson Imaging 10:49-53

12. Keller PJ, Hunter WW Jr, Schmalbrock P (1987) Multisection fat-water imaging with chemical shift selective presaturation. Radiology 164:539-541

13. Tang Y, Yamashita Y, Arakawa A, Namimoto T, Mitsuzaki K, Abe Y, Katahira K, Takahashi M (1999) Detection of hepatocellular carcinoma arising in cirrhotic livers: comparison of gadolinium- and ferumoxidesenhanced MR imaging. AJR Am J Roentgenol 172:1547-1554

14. Spencer JA, Ward J, Guthrie JA, Guillou PJ, Robinson PJ (1998) Assessment of resectability of pancreatic cancer with dynamic contrastenhanced MR imaging: technique, surgical correlation and patient outcome. Eur Radiol 8:23-29

15. Rubin DA, Kneeland JB (1994) MR imaging of the musculoskeletal system: technical considerations for enhancing image quality and diagnostic yield. AJR Am J Roentgenol 163:1155-1163
16. Bobman SA, Atlas SW, Listerud J, Grossman RI (1991) Postoperative lumbar spine: contrast-enhanced chemical shift MR imaging. Radiology 179:557-562

17. Hardy PA, Hinks RS, Tkach JA (1995) Separation of fat and water in fast spin-echo MR imaging with the threepoint Dixon technique. J Magn Reson Imaging 5:181-185

18. Glover GH, Schneider E (1991) Threepoint Dixon technique for true water/ fat decomposition with $B 0$ inhomogeneity correction. Magn Reson Med 18:371-383

19. Adam G, Bucker A, Nolte-Ernsting C, Tacke J, Gunther RW (1999) Interventional MR imaging: percutaneous abdominal and skeletal biopsies and drainages of the abdomen. Eur Radiol 9:1471-1478 